\title{
A COMPOSITION THEOREM FOR MULTIPLE SUMMING OPERATORS
}

\author{
DAVID PÉREZ-GARCÍA AND IGNACIO VILLANUEVA
}

\begin{abstract}
We prove that the composition $S\left(u_{1}, \ldots, u_{n}\right)$ of a multilinear multiple 2-summing operator $S$ with 2-summing linear operators $u_{j}$ is nuclear, generalizing a linear result of Grothendieck.
\end{abstract}

\section{InTRODUCTION AND NOTATION}

In a series of papers $([2],[9],[10],[11])$ the authors have developed the theory of multiple summing multilinear operators, generalizing the nice behavior of linear $p$-summing operators to the multilinear setting. Among other things, we have been able to obtain in the multilinear case several formulations of Grothendieck's theorem and the relations with the classes of weakly compact, integral and Hilbert-Schmidt operators. In this short note we continue showing the good behavior of this class, by proving a multilinear generalization (Theorem 2.1) of an important composition result in the linear case, namely, the result stating that the composition of two 2-summing operators is nuclear. This linear result goes back to Grothendieck [7] (see also [4] and the references therein for more about this result and its history).

First we establish our notation and recall some definitions and known facts. $X_{i}, Y$ will always be Banach spaces, and $\mathcal{L}(X, Y)$ will denote the Banach space of linear bounded mappings from $X$ to $Y$. For $n \geq 2$, $\mathcal{L}^{n}\left(X_{1} \ldots, X_{n} ; Y\right)$ will be the Banach space of all the continuous $n$-linear mappings from $X_{1} \times \cdots \times X_{n}$ into $Y$. Given a Banach space $X, X^{*}$ stands for its topological dual and $B_{X}$ denotes its unit ball. $K, K_{i}$ will stand for compact Hausdorff spaces and $C(K)$ denotes the Banach space of the continuous scalar functions defined on $K$, endowed with the supremum norm.

Next we recall the definition of several spaces of multilinear operators. We start with the definition of nuclear multilinear operator [6, Definition $1.26]$.

Definition 1.1. A multilinear operator $T: X_{1} \times \cdots \times X_{n} \longrightarrow Z$ is called nuclear if there exist sequences $\left(\phi_{i}^{j}\right)_{i=1}^{\infty} \subset X_{j}^{*}$ and $\left(z_{i}\right)_{i=1}^{\infty} \subset Z$ such that, for

2000 Mathematics Subject Classification. Primary 47H60; Secondary 46B25.

Key words and phrases. Multilinear operators, multiple $p$-summing operators, nuclear operators.

Both authors were partially supported by DGICYT grant BMF2001-1284. 
every $x_{j} \in X_{j}$

$$
T\left(x_{1}, \ldots, x_{n}\right)=\sum_{i=1}^{\infty} \phi_{i}^{1}\left(x_{1}\right) \cdots \phi_{i}^{n}\left(x_{n}\right) z_{i}
$$

and such that

$$
\sum_{i=1}^{\infty}\left\|\phi_{i}^{1}\right\| \cdots\left\|\phi_{i}^{n}\right\|\left\|z_{i}\right\|<\infty .
$$

We write $\nu(T)$ for the infimum of the numbers (2) for all the possible representations (1).

We write $\mathcal{N}^{n}\left(X_{1}, \ldots, X_{n} ; Z\right)$ for the space of nuclear multilinear operators. This space is easily seen to be a Banach space with the norm $\nu$.

The following two elementary properties of nuclear multilinear operators, needed later, are easy to prove.

Proposition 1.2. A multilinear operator $T: X_{1} \cdots \times X_{n} \longrightarrow Z$ is nuclear if and only if its associated linear operator $T_{1}: X_{1} \longrightarrow \mathcal{L}^{n-1}\left(X_{2}, \ldots, X_{n} ; Z\right)$ belongs to

$$
\mathcal{N}\left(X_{1}, \mathcal{N}^{n-1}\left(X_{2}, \ldots, X_{n} ; Z\right)\right)
$$

Moreover, in this case $\nu(T)=\nu\left(T_{1}\right)$.

Proposition 1.3. Let $u_{j} \in \mathcal{L}\left(X_{j}, Y_{j}\right)$ and $S \in \mathcal{N}^{n}\left(Y_{1}, \ldots, Y_{n} ; Z\right)$. If we define $T=S\left(u_{1}, \ldots, u_{n}\right)$, we have that $T \in \mathcal{N}^{n}\left(X_{1}, \ldots, X_{n} ; Z\right)$ and $\nu(T) \leq$ $\nu(S) \prod_{j=1}^{n}\left\|u_{j}\right\|$.

We also need the definition of integral multilinear operators. This definition appeared for the first time in [12], but it is just a technical modification of a definition given in [1].

Definition 1.4. A multilinear operator $T \in \mathcal{L}^{n}\left(X_{1}, \ldots, X_{n} ; Y\right)$ is called integral if there exists a regular Borel measure $G$ with bounded variation $v(G)<\infty$ defined on $B_{X_{1}^{*}} \times \cdots \times B_{X_{n}^{*}}$ and taking values in $Y^{* *}$ such that

$$
T\left(x_{1}, \ldots, x_{n}\right)=\int_{B_{X_{1}^{*} \times \cdots \times B_{X_{n}^{*}}}} x_{1}^{*}\left(x_{1}\right) \cdots x_{n}^{*}\left(x_{n}\right) d G\left(x_{1}^{*}, \ldots, x_{n}^{*}\right)
$$

for every $\left(x_{1}, \ldots, x_{n}\right) \in X_{1} \times \cdots X_{n}$.

The space of integral multilinear operators $\mathcal{I}^{n}\left(X_{1}, \ldots, X_{n} ; Y\right)$ is a Banach space with the norm $\iota(T)=\inf \{v(G)$, where $G$ represents $T$ as above $\}$.

We will need the following result from [12],

Proposition 1.5. The space $\mathcal{I}^{n}\left(C\left(K_{1}\right), \ldots, C\left(K_{n}\right) ; Z\right)$ is canonically isometric to $\mathcal{I}\left(C\left(K_{1}\right), \mathcal{I}^{n-1}\left(C\left(K_{2}\right), \ldots, C\left(K_{n}\right) ; Z\right)\right)$, for $K_{1}, \ldots, K_{n}$ compact Hausdorff spaces. 
To finish this section, we recall the definition of multiple $p$-summing operators, which was stated for the first time in [2] and, independently, in [8]. First we remind the reader that, for a finite sequence $\left(x_{i}\right)_{i=1}^{m} \subset X$ and $1 \leq p<\infty$, we will write $\left\|\left(x_{i}\right)_{i=1}^{m}\right\|_{p}^{\omega}$ to denote

$$
\sup \left\{\left(\sum_{i=1}^{m}\left|x^{*}\left(x_{i}\right)\right|^{p}\right)^{\frac{1}{p}}: x^{*} \in B_{X^{*}}\right\} .
$$

Definition 1.6. Let $1 \leq p<+\infty$. A multilinear operator $T: X_{1} \times \cdots \times$ $X_{n} \longrightarrow Y$ is multiple $p$-summing if there exists a constant $K>0$ such that, for every choice of sequences $\left(x_{i_{j}}^{j}\right)_{i_{j}=1}^{m_{j}} \subset X_{j}$, the following relation holds

$$
\left(\sum_{i_{1}, \ldots, i_{n}=1}^{m_{1}, \ldots, m_{n}}\left\|T\left(x_{i_{1}}^{1}, \ldots, x_{i_{n}}^{n}\right)\right\|^{p}\right)^{\frac{1}{p}} \leq K \prod_{j=1}^{n}\left\|\left(x_{i_{j}}^{j}\right)_{i_{j}=1}^{m_{j}}\right\|_{p}^{\omega}
$$

In that case, we define the multiple p-summing norm of $T$ by $\pi_{p}(T)=$ $\min \{K: K$ verifies (3) $\}$

The space $\Pi_{p}^{n}\left(X_{1}, \ldots, X_{n} ; Y\right)$ of multiple $p$-summing multilinear operators is easily seen to be a Banach space with its norm $\pi_{p}$.

\section{THE RESUlT}

Our main result is the following.

Theorem 2.1. Let $n \in \mathbb{N}$, let $X_{1}, \ldots, X_{n}, Y_{1}, \ldots, Y_{n}, Z$ be Banach spaces, let, for every $1 \leq j \leq n, u_{j}: X_{j} \longrightarrow Y_{j}$ be a 2-summing operator and let $S: Y_{1} \times \cdots \times Y_{n} \longrightarrow Z$ be a multiple 2-summing multilinear operator. Then $T=S\left(u_{1}, \ldots, u_{n}\right): X_{1} \times \cdots \times X_{n} \longrightarrow Z$ is nuclear and $\nu(T) \leq$ $\pi_{2}(S) \prod_{j=1}^{n} \pi_{2}\left(u_{j}\right)$.

The proof will rely on the following weaker version of this same result, proved in [11].

Proposition 2.2. Under the same conditions as above, $T=S\left(u_{1}, \ldots, u_{n}\right)$ : $X_{1} \times \cdots \times X_{n} \longrightarrow Z$ is integral, and $\iota(T) \leq \pi_{2}(S) \prod_{j=1}^{n} \pi_{2}\left(u_{j}\right)$.

We start proving a lemma, crucial for the proof of Theorem 2.1.

Lemma 2.3. If $K_{2}, \ldots, K_{n}$ are compact Hausdorff spaces, we have that the natural inclusion

$$
\left.\left.\mathcal{N}^{n-1}\left(C\left(K_{2}\right), \ldots, C\left(K_{n}\right) ; Z\right)\right) \hookrightarrow \mathcal{I}^{n-1}\left(C\left(K_{2}\right), \ldots, C\left(K_{n}\right) ; Z\right)\right)
$$

is an isometry.

Proof. The case $n=2$ follows from [3, 16.3] together with the fact that $C(K)^{*}$, being an $L_{1}(\mu)$ space, has the metric approximation property (see, 
for instance, [5, pág 245]). For the general case we apply induction on $n$. Let us suppose the result true for $n-2$. Using again $[3,16.3]$ we have that

$$
\begin{aligned}
& \mathcal{N}\left(C\left(K_{2}\right), \mathcal{N}^{n-2}\left(C\left(K_{3}\right), \ldots, C\left(K_{n}\right) ; Z\right)\right) \stackrel{1}{\hookrightarrow} \\
& \stackrel{1}{\hookrightarrow} \mathcal{I}\left(C\left(K_{2}\right), \mathcal{N}^{n-2}\left(C\left(K_{3}\right), \ldots, C\left(K_{n}\right) ; Z\right)\right),
\end{aligned}
$$

where the sign $\stackrel{1}{\hookrightarrow}$ means that the natural inclusion is an isometry.

Now, the induction hypothesis implies that

$$
\mathcal{N}^{n-2}\left(C\left(K_{3}\right), \ldots, C\left(K_{n}\right) ; Z\right) \stackrel{1}{\hookrightarrow} \mathcal{I}^{n-2}\left(C\left(K_{3}\right), \ldots, C\left(K_{n}\right) ; Z\right) .
$$

Since integral linear operators defined on a $C(K)$ coincide with 1-summing operators [4, Corollary 5.8], and using the fact that 1-summing operators form an injective operator ideal [3, 11.1], we get that

$$
\begin{aligned}
& \mathcal{I}\left(C\left(K_{2}\right), \mathcal{N}^{n-2}\left(C\left(K_{3}\right), \ldots, C\left(K_{n}\right) ; Z\right)\right) \stackrel{1}{\hookrightarrow} \\
& \stackrel{1}{\hookrightarrow} \mathcal{I}\left(C\left(K_{2}\right), \mathcal{I}^{n-2}\left(C\left(K_{3}\right), \ldots, C\left(K_{n}\right) ; Z\right)\right) .
\end{aligned}
$$

Propositions 1.5 and 1.2 suffice to finish the proof.

We will also need trace duality, as it appears in the next result, which follows from [3, 25.7] and [4, Corollary 6.17].

Proposition 2.4. A linear operator $u: Y \longrightarrow Z$ is 2-summing if and only if, for every 2-summing operator $v: X \longrightarrow Y, u v: X \longrightarrow Z$ is integral. Moreover,

$$
\pi_{2}(u)=\sup \left\{\iota(u v): \pi_{2}(v) \leq 1\right\} .
$$

We can finally prove our main result.

Proof of Theorem 2.1. We apply induction on $n$. The case $n=1$ is well known [4, Theorem 5.31].

Let us suppose the result true for $n-1$, and let us note that, using Pietsch's Factorization Theorem [4, Corollary 2.16] and Proposition 1.3, we can assume without loss of generality that $X_{2}=C\left(K_{2}\right), \ldots, X_{n}=C\left(K_{n}\right)$ for certain compact Hausdorff spaces $K_{2}, \ldots, K_{n}$.

The induction hypothesis allows us to define the operator

$$
u: \Pi_{2}^{n-1}\left(Y_{2}, \ldots, Y_{n} ; Z\right) \longrightarrow \mathcal{N}^{n-1}\left(C\left(K_{2}\right), \ldots, C\left(K_{n}\right) ; Z\right)
$$

by $u(R)=R\left(u_{2}, \ldots, u_{n}\right)$.

If $S_{1}: Y_{1} \longrightarrow \Pi_{2}^{n-1}\left(Y_{2}, \ldots, Y_{n} ; Z\right)$ is the linear operator associated to $S$, we define $w=u S_{1}: Y_{1} \longrightarrow \mathcal{N}^{n-1}\left(C\left(K_{2}\right), \ldots, C\left(K_{n}\right) ; Z\right)$.

We use now the following claim, which we prove below.

Claim: $w$ is 2 -summing and $\pi_{2}(w) \leq \pi_{2}(S) \prod_{j=2}^{n} \pi_{2}\left(u_{j}\right)$.

Then, it follows from the case $n=1$ that the linear operator associated to $T, T_{1}: X_{1} \longrightarrow \mathcal{N}^{n-1}\left(C\left(K_{2}\right), \ldots, C\left(K_{n}\right) ; Z\right)$, which is exactly $T_{1}=w u_{1}$, is nuclear and $\nu\left(T_{1}\right) \leq \pi_{2}(S) \prod_{j=1}^{n} \pi_{2}\left(u_{j}\right)$. We can use now Proposition 1.2 to conclude that $T$ is nuclear and $\nu(T) \leq \pi_{2}(S) \prod_{j=1}^{n} \pi_{2}\left(u_{j}\right)$. 
The proof will be completely finished when we prove our Claim. It follows from Proposition 2.4 that it is enough to check that, for every 2-summing operator $v: V \longrightarrow Y_{1}, w v$ is integral and $\iota(w v) \leq \pi_{2}(v) \pi_{2}(S) \prod_{j=2}^{n} \pi_{2}\left(u_{j}\right)$.

So let us consider one such $v$. Using again Pietsch's Factorization Theorem and Lemma 2.3, we have the following diagram

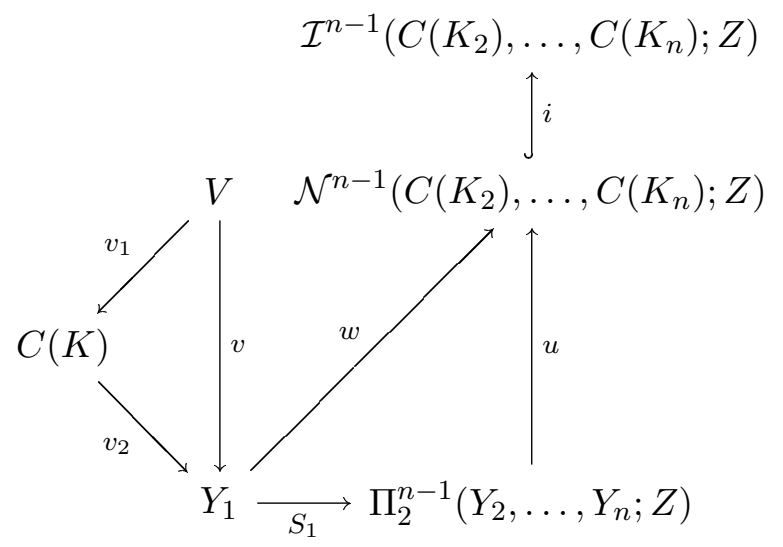

where $i$ is an isometric inclusion, $\left\|v_{1}\right\|=1$ and $\pi_{2}\left(v_{2}\right)=\pi_{2}(v)$. According to Theorem 2.2 and Proposition 1.5, we know that $i w v_{2}$ is integral and $\iota\left(i w v_{2}\right) \leq \pi_{2}(v) \pi_{2}(S) \prod_{j=2}^{n} \pi_{2}\left(u_{j}\right)$. As mentioned in the introduction, integral operators coincide with 1 -summing operators when defined on a $C(K)$ space, and 1-summing operators form an injective operator ideal. Therefore $w v_{2}$, and hence $w v$, are integral operators and

$$
\iota(w v) \leq\left\|v_{1}\right\| \iota\left(w v_{2}\right) \leq \pi_{2}(v) \pi_{2}(S) \prod_{j=2}^{n} \pi_{2}\left(u_{j}\right),
$$

which proves the claim and finishes the proof of the theorem.

\section{REFERENCES}

[1] R. Alencar, Multilinear mappings of nuclear and integral type, Proc. Amer. Math. Soc. 94 (1985), no. 1, 33-38.

[2] F. Bombal, D. Pérez-García, and I. Villanueva, Multilinear extensions of Grothendieck's theorem, to appear in Q. J. Math.

[3] A. Defant and K. Floret, Tensor norms and operator ideals, North-Holland, 1993.

[4] J. Diestel, H. Jarchow, and A. Tonge, Absolutely summing operators, Cambridge Univ. Press, 1995.

[5] J. Diestel and J.J. Uhl, Vector measures, Mathematical Surveys and Monographs, no. 15, Amer. Math. Soc., 1977.

[6] S. Dineen, Complex analysis in locally convex spaces, North-Holland, 1981.

[7] A. Grothendieck, Produits tensoriels topologiques et espaces nucléaires, Mem. Amer. Math. Soc. 16 (1955).

[8] M.C. Matos, Fully absolutely summing and Hilbert-Schmidt multilinear mappings, Collect. Math. 54 (2003), 111-136.

[9] D. Pérez-García, The inclusion theorem for multiple summing operators, Preprint.

[10] D. Pérez-García and I. Villanueva, Multiple summing operators on Banach spaces, J. Math. Anal. Appl. 285 (2003), 86-96. 
[11] D. Pérez-García and I. Villanueva, Multiple summing operators on $C(K)$ spaces, To appear in Ark. Mat.

[12] I. Villanueva, Integral mappings between Banach spaces, J. Math. Anal. Appl. 279 (2003), 56-70.

E-mail address: David_Perez@mat.ucm.es, ignaciov@mat.ucm.es

Departamento de Análisis Matemático, Facultad de Matemáticas, UniverSidAd Complutense De Madrid, Madrid 28040 